\title{
Original Article (short paper) \\ Effects of previous carbohydrate supplementation on muscular fatigue: double-blind, randomized, placebo-controlled crossover study
}

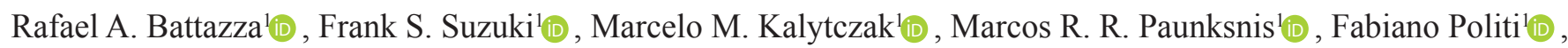

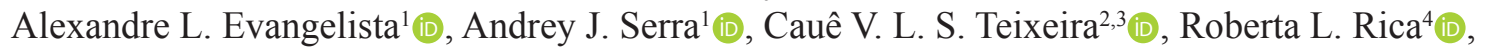 \\ Francisco Luciano Pontes Junior ${ }^{5}$, Marco Bergamin ${ }^{6}$ (D), Danilo S. Bocalini ${ }^{7}$ (D) \\ ${ }^{1}$ Universidade Nove de Julho, UNINOVE, Sao Paulo, SP, Brazil; ${ }^{2}$ Universidade Federal de Sao Paulo, UNIFESP, \\ Department of Biosciences, Sao Paulo, SP, Brazil, ${ }^{3}$ Faculdade Praia Grande, Faculdade de Educacao Fisica, \\ Praia Grande, SP, Brazil; ${ }^{4}$ Universidade Estacio de Sá, Vitoria, ES, Brazil; ${ }^{5}$ Universidade de Sao Paulo, USP, Es- \\ cola de Artes Ciencias e Humanidades, Sao Paulo, SP, Brazil; ' ${ }^{-}$Universita degli Studi di Padova, Dipartimen- \\ to di Medicina, Padova, Veneto, Italia; ${ }^{7}$ Universidade Federal do Espirito Santos, UFES, Vitoria, ES, Brazil
}

\begin{abstract}
Aims: The aim of this study was to examine the effects of previous carbohydrate supplementation on high-volume resistance exercise performance. Methods: Twenty males physically independent adults aged $\geq 18$ years participated in a double-blind randomized placebo-controlled crossover study. Sixty minutes before the experimental protocol, each participant ingested 0,6 g.kg of body mass ${ }^{-1}$ of carbohydrate supplementation or placebo. Maximum voluntary isometric contraction tests were performed before and after the dynamic fatigue induction protocol consisting of 10 sets of 8 repetitions of right leg knee extensors at $120^{\circ} \mathrm{s}^{-1}$. Results: Lower decrement of the isometric peak torque $(p<0,001)$ and of the rate of torque development $(p<0,001)$ was observed in carbohydrate supplementation after the dynamic protocol. Both concentric and eccentric peak torque differed significantly $(p<0,001)$ between carbohydrate supplementation and placebo treatments from the second set, although the slope of the force-repetitions curve was not different between them. Additionally, the carbohydrate supplementation resulted in a lower session rating of perceived exertion $(\mathrm{p}<0,05)$. Conclusions: Previous carbohydrate supplementation attenuates muscle fatigue and internal load exercise in a high-volume isokinetic leg protocol.
\end{abstract}

Keywords: carbohydrate ingestion; resistance exercise; performance; training load

\section{Introduction}

Reductions in muscle glycogen content have been shown to compromise resistance exercise (RE) performance ${ }^{1-3}$, typically performed by short-term contractions of relatively high intensity against an external resistance ${ }^{4}$. Carbohydrate supplementation (CS) attenuates reductions in muscle glycogen content during $\mathrm{RE}$, which could result in improved performance ${ }^{5}$. However, the data in the literature are conflicting ${ }^{5-9}$ which causes uncertainty regarding the efficacy of $\mathrm{CS}$ as an ergogenic aid in this type of exercise.

Haff et al. ${ }^{3}$ observed during the second session of a training day a significant improvement of multiple sets of squat exercise performance to exhaustion at $55 \%$ of one repetition maximum (RM) when CS was ingested by the participants compared to placebo. Conversely, no improvement was observed in squat at $85 \%$ of $1 \mathrm{RM}$ until voluntary failure with $\mathrm{CS}^{9}$. Additional investigation ${ }^{8}$ have reported no significant alteration in the number of repetitions (performance evaluation) in a bout with seven REs performed at $70 \%$ of $1 \mathrm{RM}$ when resistance-trained individuals ingested CS or placebo.

The effects of CS on isokinetic exercise performance are also contradictory ${ }^{5,7,10}$. Previous research ${ }^{7}$ indicate that the use of this supplement during high-volume isokinetic exercise allows more total work. However, the CS prior to a free-weight RE bout did not result in improved performance of subsequent isokinetic exercise $^{10}$. Likewise, no effect of CS on isokinetic exercise performed after three isotonic RE bouts was reported although significantly lower glycogen degradation was observed in the CS condition compared to the placebo ${ }^{5}$.

These contrasting results may be related to different exercise protocols and the supplementation employed, as well as the type of exercise used (isokinetic or isotonic) ${ }^{11}$. Furthermore, the duration of exercise seems to be an important factor for the potential ergogenic effect of CS to occur, since protocols with durations longer than 40 minutes could result in a greater volume of exercise generating greater stress of the glycolytic system ${ }^{11,12}$ with greater decreases in muscle glycogen content occur when high volume training with moderate loads is employed ${ }^{13}$.

It is also interesting to note that in the majority of cases, these investigations ${ }^{7-10}$ have focused on the effect of this supplement on external load parameters, with little information on the effect of CS on internal load parameters, for example, the rate of perceived exertion ${ }^{14,15}$.

To investigate the effect of prior carbohydrate supplementation on both external load and internal load parameters on $\mathrm{RE}$, further investigations are required and the use of isokinetic dynamometer may help in understanding the effects of 
supplementation on specific muscle groups (and not only on specific exercises) and on conditions related to muscle fatigue with accuracy, once the isokinetic testing has been regarded as a principal method to evaluate muscle function ${ }^{16}$. Thus, the aim of the present study was to examine the effects of previous carbohydrate supplementation on the performance of high-volume isokinetic leg exercise as well as the internal load parameter assessed through the session rate of perceived exertion.

\section{Methods}

\section{Participants}

Twenty male physically independent adults aged $\geq 18$ years voluntarily participated in this study. Subjects with diabetes mellitus, current smokers, individuals with musculoskeletal injuries and diagnosed clinical pathologies and/or cardiovascular changes confirmed by medical evaluation and ingestion of any other dietary supplements for a minimum of 12 weeks before the initiation of this study were excluded.

This study was conducted according to the guidelines laid down in the Declaration of Helsinki and all procedures involving human subjects were approved by the Research Ethics Committee of the Sao Judas Tadeu University, number 786.06-2014. Written informed consent was obtained from all subjects.

\section{Anthropometric measurements}

Anthropometric assessment was performed following the model previously outlined by our group ${ }^{17}$. Briefly, height was measured with a Cardiomed stadiometer (WCS model), with a $115 / 120 \mathrm{~cm}$ range. The measurement was performed with the cursor placed at a $90^{\circ}$ angle with the scale in the upright position, and feet placed together in contact with the stadiometer. Subjects were instructed to hold their breath and keep the head parallel to the ground. Weight was measured with a calibrated electronic scale (Filizola - Personal Line Model 150), with a range of $100 \mathrm{~g}$, and maximum capacity of $150 \mathrm{~kg}$. Body mass index was calculated and recorded from the equation $\mathrm{kg} / \mathrm{cm}^{2}$.

\section{Procedures}

\section{Carbohydrate supplementation}

All participants were instructed to eat as they normally would in preparation for a fatigue protocol and to replicate the same dietary strategy before each trial. Participants recorded their intake on the day leading up to each trial. All subjects presented adequate energy intake in agreement to according to previously recommendations ${ }^{18}$.

This was a double blind, randomized, placebo-controlled crossover study. During the study, the participants were asked to maintain their dietary patterns and abstain from other nutritional supplements, nonprescription drugs, and caffeine. Prior to the testing, each participant was randomly assigned to either the placebo or carbohydrate (maltodextrin) supplementation (CS) approach. The carbohydrate supplementation $(6 \%-60 \mathrm{~g}$ of maltodextrin. $\left.\mathrm{L}^{-1}\right)$ was adapted from the previous studies ${ }^{5,19}$. The placebo or CS were the same color, flavor, and consistency, and were administered for all groups and administered in water $(500 \mathrm{ml}) 60$ minutes before the fatigue protocol.

\section{Fatigue induction protocol}

The experimental fatigue protocol design is according to previous publications of our group ${ }^{17}$. Briefly, all subjects attended in the laboratory four times to perform the isokinetic evaluation by using a Biodex System 3 (Biodex, Inc., Shirley, $\mathrm{NY}$ ) at the same time in all experimental condition (from 14 to $16 \mathrm{pm}$ ). The validity coefficient of this instrument is 0.99 , and the reproducibility measuring peak torque during knee extension was 0.92 at younger's subjects. On the first day, the subjects signed consent forms and completed the International Physical Activity Questionnaires (IPAQ). On the second day, the anthropometric data (height, and weight) was evaluated. On the third and fourth day, the subjects were placed on the Isokinetic Dynamometer in a comfortable, upright, seated position for familiarization procedures. Straps were used to fix the thigh, pelvis, and trunk to prevent extraneous body movement. The axis of the dynamometer was aligned with the axis of right knee joint rotation. Arms were placed across the chest with hands grasping the straps.

The experimental protocol consisted of tests of maximum voluntary isometric contraction (MVIC) and dynamic protocol. The MVIC tests were performed before (pre-MVIC test) and after (post-MVIC test) the dynamic protocol and consisted of the execution of three maximal isometric contractions of the dominant leg knee extensors with duration of five seconds and five minutes of recovery intervals between contractions. Five minutes recovery intervals separated the MVIC tests from the dynamic protocol. The determination of the MVIC of the knee extensor muscles was performed on the two days of intervention. For the isometric tests, the participant's knee was positioned at an angle of $60^{\circ}$, considering the $0^{\circ}$ point a total knee extension.

For the dynamic protocol, ten sets of eight repetitions of concentric knee extension/flexion at $120^{\circ} . \mathrm{s}^{-1}$ were performed. The knee joint range of motion was 90 to $10^{\circ}$ of knee flexion. The calibration of the Isokinetic Dynamometer was performed according to the specifications of the manufacturer before each test, and finally, on the fourth day, the fatigue protocol was performed. All testing and experimental procedures were performed at the same time of day to exclude diurnal variation.

\section{Perceived exertion and training load evaluation}

In both experimental treatments, session rate of perceived exertion (internal load) in arbitrary units was calculated by multiplying the duration of the session by perceived exertion (CR-10 scale) evaluated 30 minutes after the end of the session ${ }^{20}$. 


\section{Statistical analysis}

The analyses were performed using the IBM SPSS Statistics version 21.0 (SPSS, Chicago, IL, USA). The D'AgostinoPearson was used to test the normality of the data distribution. The Student's $t$-test was used to compare the variables of pre e post MVIC test. The two-way repeated-measures analysis of variance (ANOVA) design followed by a post-hoc Bonferroni was used to compare group (CS and placebo) vs sets (multiple comparisons). The effect sizes (ES) were calculated, and evaluated based on the following criteria proposed by $\mathrm{Rhea}^{21}:<0.50$ trivial, 0.50 to 1.25 small, 1.25 to 1.9 moderate and $>2$ large.
Data are expressed as the mean and standard error of the mean, and the significance level was set at $\mathrm{p}<0.05$.

\section{Results}

No participants experienced any injuries before, during or after both fatigue protocols. The biometric and physical activity level parameters assessed are described in Table 1. No difference ( $>0.05)$ was found on energy intake independently of experimental condition (No Carbo: $2680 \pm 340$, Carbo: 2750 \pm 480 ; Kcal)

Table 1. Sample characteristics.

\begin{tabular}{lcc}
\hline \multicolumn{1}{c}{ Parameters } & Mean \pm SD & 95\% of IC \\
\hline Age (years) & $25.13 \pm 4.35$ & $21.48-28.77$ \\
Body mass (kg) & $76.25 \pm 7.59$ & $69.90-82.60$ \\
Height (cm) & $1.77 \pm 0.06$ & $1.72-1.83$ \\
BMI (kg/cm2) & $24.00 \pm 1.06$ & $23.11-24.89$ \\
Physical activity level (min/week) & $309.83 \pm 1.06$ & $239.90-367.90$ \\
\hline
\end{tabular}

Values are presented as mean \pm standard deviation. BMI: body mass index.

As showed in Figure 1 a significant difference $(\mathrm{p}<0.001)$ was observed in the isometric peak torque with CS prior to the fatigue protocol. After the fatigue protocol, significant reductions $(\mathrm{p}<0.001)$ were found in both isometric peak torque and rate of torque development after the CS and placebo approaches. However, placebo presented a greater $(\mathrm{p}<0.001)$ impairment in isometric peak torque (before: $321 \pm 74 \mathrm{Nm}$, after: $144 \pm 57$ $\mathrm{Nm}$ ) and in rate of torque development (before: $137 \pm 54 \mathrm{Nm} / \mathrm{s}$, after: $33 \pm 13 \mathrm{Nm} / \mathrm{s}$ ) compared to CS treatment, isometric peak torque (before: $343 \pm 68 \mathrm{Nm}$, after: $238 \pm 61 \mathrm{Nm}$ ) and rate of torque development (before: $137 \pm 51 \mathrm{Nm} / \mathrm{s}$, after: $67 \pm 24 \mathrm{Nm} / \mathrm{s}$ ). No significant interactions were found on isometric peak torque (interaction: $17.62, \mathrm{~F}: 0.6450, \mathrm{p}=0.7134$ ) and rate of torque development up from the chair test and arm flexion (Interaction: 10.20, F: 0.3351, $\mathrm{p}=0.9261$ ). The analysis of the effect size and both isometric peak torque and rate of torque development parameters of placebo $(2.39,1.92)$ and CS $(1.54,1.37)$ presented large effects, however, the alterations of placebo were more evident.
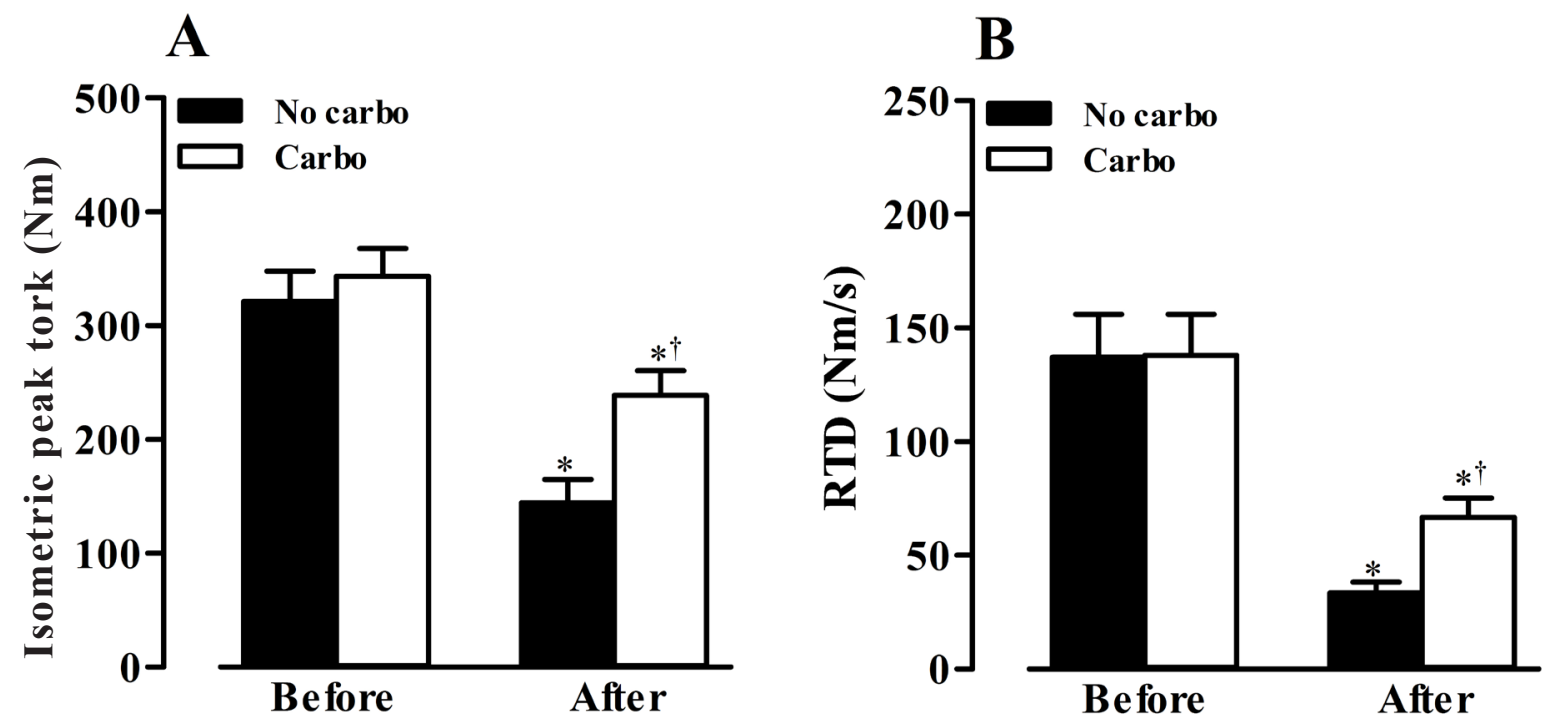

Figure 1. Values expressed in mean $\pm \mathrm{SD}$ of isometric peak torque (panel A) and rate of torque development (Panel B) before and after fatigue induction protocol after placebo or carbohydrate supplementation intake.

* significantly different from before $(\mathrm{p}=0.01)$.

$\uparrow$ significantly different from no carbo $(\mathrm{p}=0.01)$. 
Significant differences $(\mathrm{p}<0.001)$ were found in the absolute torque in both $\mathrm{CS}$ and placebo after the second set in both concentric (Figure 2A) and eccentric (Figure 2C) muscular action. Significant differences $(p<0.001)$ were observed in the peak torque between the treatments, with and without CS from the second set in both concentric (Interaction: 39.81, F: 20.10, p= 0.004 ) and eccentric (Interaction: 17.42, F: 4.766, $p=0.0443$ ) actions. However, there were no significant differences $(\mathrm{p}>0.05)$ in the slope of the force-repetition curve in either the concentric (placebo: $-10.67 \pm 1.97$; CS: $-7.44 \pm 4.58$; Nm/set) or eccentric action (placebo: $-15.24 \pm 7.82$; CS: $-12.28 \pm 4.45$; Nm/set). In addition, no differences were found between the muscle action lines independent of the CS.

A significant percentage reduction $(p<0.001)$ was found after 10 sets in the maintenance of concentric and eccentric torque, respectively, in both the placebo (concentric: $-43 \pm 9$, eccentric: $-49 \pm 17$ ) and CS (concentric: $-23 \pm 11$; eccentric: $-35 \pm 12$ ) as shown in figures $2 \mathrm{~B}$ and $2 \mathrm{D}$. The analysis of the effect size of both muscle action of peak torque of placebo $(3.36,2.91)$ and with CS $(1.17,2.18)$ presented large effect; however, such as isometric peak torque, the alterations of CS were more evident.
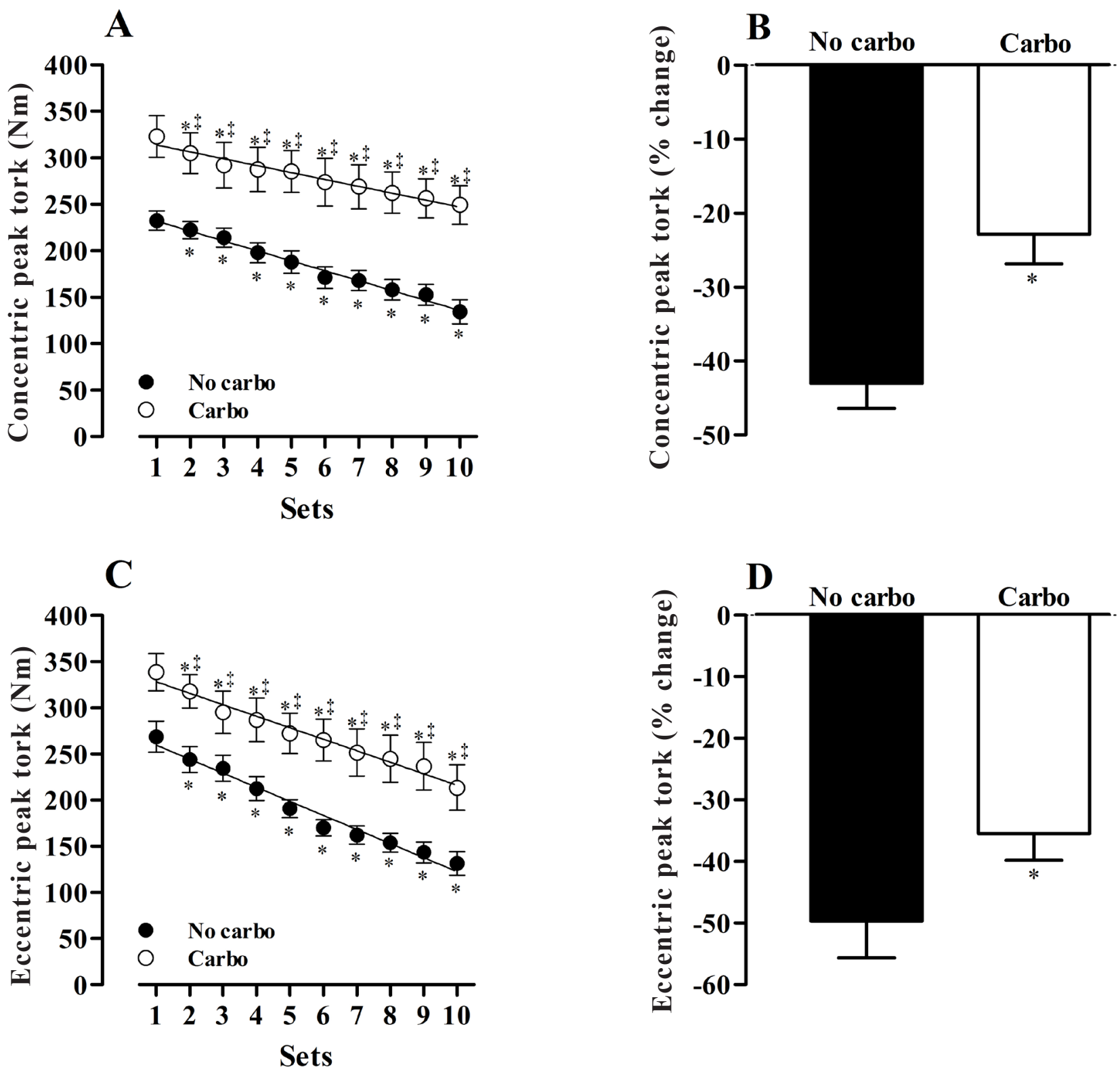

Figure 2. Values expressed in mean $\pm \mathrm{SD}$ of decline of concentric (panel A) and eccentric peak torque (panel C) during 10 sets of isokinetic mode knee extensions at $120^{\circ} \mathrm{sec}^{-1}$ and perceptual changes of concentric (panel B) and eccentric peak torque (panel D) after fatigue induction after placebo or carbohydrate supplementation intake.

*significantly different from $1^{\mathrm{a}}$ set $(\mathrm{p}=0.01)$.

†significantly different from no carbo $(\mathrm{p}=0.01)$. 
Additionally, as shown in figure 3 , the CS present less $(\mathrm{p}<0.05)$ internal load (787 $\pm 83 \mathrm{au})$ compared to placebo $(950 \pm 75 \mathrm{au})$.

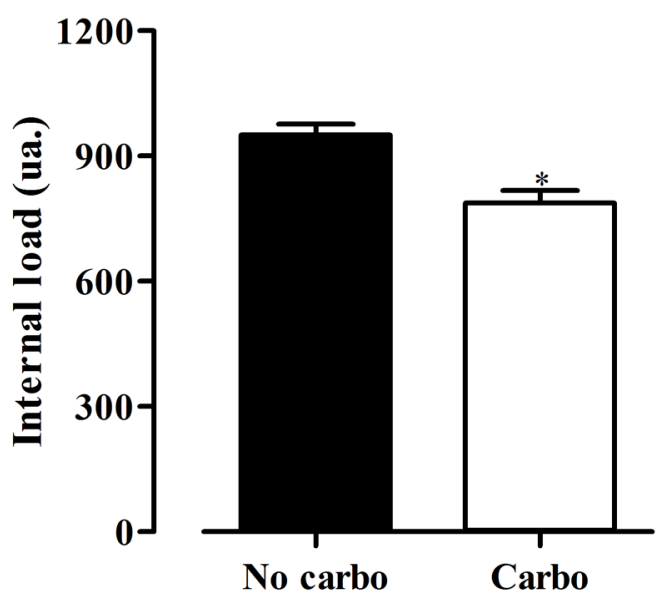

Figure 3. Values expressed in mean \pm SD of internal load in arbitrary units evaluated 30 minutes after the end of the session after placebo or carbohydrate supplementation intake. au: Arbitrary units

*significantly different from no carbo $(\mathrm{p}=0.005)$.

\section{Discussion}

The purpose of the present study was to examine the effects of previous carbohydrate supplementation on high-volume isokinetic leg exercise performance. Two important points should be addressed to clarify this study. First, according to Hofman, Van Buul, Brouns ${ }^{22}$ the maltodextrins was used in the present study due to fast digestibility and absorption in the small intestine and subsequently used in metabolism. Second, although isokinetic actions are not common everyday actions, the use of isokinetic dynamometry allows the continuous monitoring of external load of exercise with greater accuracy of musculoskeletal performance ${ }^{16}$.

As expected, the fatigue protocol resulted in significant decrease in concentric and eccentric peak torque values, with or without carbohydrate supplementation. However, the main finding of this study was that both concentric and eccentric peaks brought about an attenuation of the fatigue process after the CS compared with placebo.

The high-volume moderate-load ERs are partially dependent on the muscular glycogen store, with several investigations ${ }^{1,2}$ showing a reduction in the content of this substrate after multiple sets of ERs and, therefore, the improvement of the performance we observed may have occurred due to the smaller reduction of muscle glycogen content after $\mathrm{CS}^{5}$.

Additionally, the ergogenic effect of supplementation may also have occurred due to a lower rate of glycogenolysis resulting from increased glycogen synthesis during the rest intervals between sets $^{5,23}$ since the protocol consisted of 10 sets with 45 seconds of rest between them. However, it is important to note that the content of this substrate was not evaluated in the present investigation.
The data about the effect of carbohydrate supplementation on neuromuscular performance using the isokinetic evaluation are contradictory and inconclusive $e^{5,7,10}$. Although the use of isokinetic dynamometer may help in understanding the conditions related to muscle fatigue with accuracy allowing to verify some muscular characteristics as the torque ${ }^{16}$, in isokinetic exercise the force is only applied during a relatively small range of motion, which can result in a decreased amount of work (compared with isotonic exercise) and mask the potential ergogenic effect of supplementation ${ }^{24}$. In addition, the discrepancies found in both isokinetic protocols and conventional RE (e.g., free-weights) ${ }^{6,8,9}$ can be attributed to the total duration of the session since the amount of glycogen utilization seems to be influenced by the duration of the protocol. Indeed, a greater ergogenic effect in response to CS occurs in protocols of longer duration ${ }^{12}$.

However, our findings challenge this premise because the ergogenic effect of supplementation was demonstrated even with an isokinetic exercise protocol with a total duration of only 29 minutes. Previous studies have showed improvement in performance after approximately 57 minutes $^{7}$ and 56 minutes ${ }^{6}$ whereas another study ${ }^{25}$ failed to find an improvement in exercise after approximately 35 minutes.

Consistent with our findings, Haff, Schroeder, Koch, Kuphal, Comeau, Potteiger ${ }^{7}$ also observed improvement in the performance of isokinetic leg exercise with CS. In the study in question, a significantly greater amount of work was observed during the performance of 16 sets of 10 repetitions at $120^{\circ} \mathrm{s}^{-1}$, in knee extension and flexion with CS compared with placebo. In contrast with the present study, the supplementation protocol in Haff, Schroeder, Koch, Kuphal, Comeau, Potteiger ${ }^{7}$ was performed both before and during exercise, and as mentioned, exercise duration was longer $(57 \mathrm{~min})$. Thus, it is possible that the ergogenic effect of CS may be associated with the high volume of exercise performed, and not necessarily the duration of the effort or the supplementation mode.

In the present investigation, the MVIC test was performed before and after the dynamic fatigue protocol. The concentric and eccentric peak torque values did not differ in the MVIC pre-dynamic protocol between treatments, indicating that the isometric strength and the ability to produce maximum activation in the initial phases (rate of torque development) of the exercise were not affected by the supplementation. The short duration of effort in the MVIC test may explain the results observed, since most of the energy required for short-term muscle contraction is available from the ATP-CP pathway and not from the glycolytic system, and therefore the muscle glycogen content is not significantly reduced ${ }^{1}$. However, after the dynamic protocol, both concentric and eccentric peak torques were less compromised when subjects were supplemented with carbohydrate, demonstrating the ergogenic effect of CS.

After the dynamic protocol, it is likely that the MVIC test was performed with the greater participation of the glycolytic system and, therefore, a lower decrease of muscle glycogen content when subjects were supplemented with carbohydrate and may have resulted in lower isometric peak torque impairment. Although related to prolonged exercise, the positive effect on the central nervous system (central fatigue attenuation) associated 
with carbohydrate intake may have contributed to the higher rate of torque development in this situation ${ }^{5}$.

Compared to placebo, the CS attenuated the internal load evaluated through session rate of perceived exertion, which was indicated as a valid method to monitor the intensity of the exercise session ${ }^{20}$. Some studies have investigated the effect of $\mathrm{CS}$ on the rate of perceived exertion responses in endurance exercises ${ }^{14,26}$ with less attention given to REs ${ }^{15}$.

For example, the attenuation of the rate of perceived exertion in the final phase (last $30 \mathrm{~min})$ of prolonged running $(3 \mathrm{~h})$ on the treadmill after CS was attributed to a greater use of the blood glucose available by carbohydrate intake ${ }^{14}$. However, no relationship was found between CS and rate of perceived exertion (Borg 6-20 scale) during RE with a duration of $2 \mathrm{~h}^{15}$. The mechanisms pointed to attenuation of the rate of perceived exertion response in the endurance exercises ${ }^{15}$ are difficult to extrapolate to the REs, which could be characterized by short and intermittent efforts with greater anaerobic contribution ${ }^{4}$.

\section{Conclusions}

In conclusion, the present findings suggest that previous carbohydrate supplementation may attenuate the muscle fatigue process in a high-volume isokinetic leg exercise and result in lower session rate of perceived exertion, even with total exercise duration of fewer than 40 minutes. However, considering the small number of studies examining the effects of CS on the rate of perceived exertion in resistance training, future investigations in this area are necessary. Our data also suggest that the previous supplementation with carbohydrate is essential for better performance in REs since when individuals followed their usual diets a greater impairment in performance was observed. The potential ergogenic effect of CS can ultimately allow individuals who perform REs to train at higher intensities or do more work during daily training sessions, enhancing the physiological adaptations that are associated with resistance training.

\section{References}

1. 1. Robergs RA, Pearson DR, Costill DL, Fink WJ, Pascoe DD, Benedict MA, et al. Muscle glycogenolysis during differing intensities of weight resistance exercise. J Appl Physiol. 1991;70(4):1700-6.

2. 2. Tesch PA, Ploutz-Snyder LL, Ystrom L, Castro M, Dudley G. Skeletal muscle glycogen loss evoked by resistance exercise. J Strength Cond Res. 1998;12(2):67-73.

3. 3. Haff GG, Stone MH, Warren BJ, Keith R, Johnson RL, Nieman DC, et al. The effect of carbohydrate supplementation on multiple sessions and bouts of resistance exercise. J Strength Cond Res. 1999;13(2):111-7.

4. 4. American College of Sports Medicine. American College of Sports Medicine position stand. Progression models in resistance training for healthy adults. Med Sci Sports Exerc. 2009;41(3):687-708.
5. 5. Haff GG, Koch AJ, Potteiger JA, Kuphal KE, Magee LM, Green SB, et al. Carbohydrate supplementation attenuates muscle glycogen loss during acute bouts of resistance exercise. Int J Sport Nutr Exerc Metab. 2000;10(3):326-39.

6. 6. Lambert CP; Flynn MG. Fatigue during High-Intensity Intermittent Exercise: Application to Bodybuilding. Sports Med. 2002;32(8):511-22.

7. 7. Haff GG, Schroeder CA, Koch AJ, Kuphal KE, Comeau MJ, Potteiger JA. The effects of supplemental carbohydrate ingestion on intermittent isokinetic leg exercise. J Sports Med Phys Fitness. 2001;41(2):216-22.

8. 8. Fayh APT, Umpierre D, Sapata KB, Neto FMDN, Oliveira AR. Efeitos da ingestão prévia de carboidrato de alto índice glicêmico sobre a resposta glicêmica e desempenho durante um treino de força. Rev Bras Med Esporte. 2007;13(6):416-20.

9. 9. Kulik JR, Touchberry CD, Kawamori N, Blumbert PA, Crum AJ, Haff GG. Supplemental carbohydrate ingestion does not improve performance of high-intensity resistance exercise. J Strength Cond Res. 2008; 22(4):1101-7.

10. 10. Vincent KR, Clarkson PM, Freedson PS, Decheke M. Effects of pre-exercise liquid, high carbohydrate feeding on resistance exercise performance. Med Sci Sports Exerc. 1993;25:S194.

11. 11. Haff GG, Lehmkuhl MJ, McCoy LB, Stone MH. Carbohydrate supplementation and resistance training. J Strength Cond Res. 2003;17(1):187-96.

12. 12. Anantaraman R, Carmines AA, Gaesser GA, Weltman A. Effects of carbohydrate supplementation on performance during 1 hour of high-intensity exercise. Int J Sports Med. 1995;16(7):461-5.

13. 13. Knuiman P, Hopman MTE, Mensink M. Glycogen availability and skeletal muscle adaptations with endurance and resistance exercise. Nutr Metab (Lond). 2015;12(59):1-11.

14. 14. Utter AC, Kang J, Nieman DC, Dumke CL, McAnulty SR, Vinci DM, et al. Carbohydrate supplementation and perceived exertion during prolonged running. Med Sci Sports Exerc. 2004,36(6):1036-41.

15. 15. Utter AC, Kang J, Nieman DC, Brown VA, Dumke CL, McAnulty SR, et al. Carbohydrate supplementation and perceived exertion during resistance exercise. J Strength Cond Res. 2005;19(4):939-43.

16. 16. De Ste Croix MBA, Deighan MA, Armstrong N. Assessment and Interpretation of Isokinetic Muscle Strength During Growth and Maturation. Sports Med. 2003;33(10):727-743.

17. 17. Suzuki FS, Kalytczak MM, Casarin CAS, Serra AJ, Evangelista AL, Marchetti PH, et al. Physical activity level does not influence the neuromuscular fatigue in adults. Rev Bras Med Esporte. 2016;22(2):97-101.

18. 18. Thomas DT, Erdman KA, Burke LM. American College of Sport Medicine. Joint Positions Statement. Nutrition and Athletic Performance. Med Sci Sport Exerc. 2016;48(3):543-68.

19. 19. Aoki MS, Júnior FLP, Navarro F, Uchida MC, Bacurau RFP. Carbohydrate supplementation fails to revert the deleterious effects of endurance exercise upon subsequent strength performance. Braz J Sport Med. 2003;9(5):282-287.

20. 20. Foster C, Florhaug JA, Franklin J, Gottschall L, Hrovatin L, Parker S, et al. A new approach to monitoring exercise training. J Strength Cond Res. 2001;15(1):109-15. 
21. 21. Rhea MR. Determining the magnitude of treatment effects in strength training research through the use of effect size. J Strength Cond Res. 2004;18(4):918-920.

22. 22. Hofman DL, Van Buul VJ, Brouns FJPH. Nutrition, health, and regulatory aspects of digestible maltodextrins. Crit Rev Food Sci Nutr. 2016; 56(12):2091-2100.

23. 23. Leatt PB, Jacobs I. Effect of glucose polymer ingestion on glycogen depletion during a soccer match. Can J Sport Sci. 1989;14(2):112-6.

24. 24. Chow JW, Darling WG, Hay JG. Mechanical characteristics of knee extension exercises performed on an isokinetic dynamometer. Med Sci Sports Exerc. 1997;29(6):794-803.

25. 25. Conley M, Stone M, Marsit JL, O'Bryant HS, Nieman DC, Johnson, JL. Effects of carbohydrate ingestion on resistance exercise. J Strength Cond Res. 1995;9:20.

26. 26. Kang J, Robertson RJ, Goss FL, DaSilva SG, Visich P, Suminski RR, et al. Effect of carbohydrate substrate availability on ratings of perceived exertion during prolonged exercise of moderate intensity. Percept Mot Skills.1996;82(2):495-506.

\section{Corresponding author}

Danilo Sales Bocalini

Universidade Federal do Espirito Santo, Goiabeiras, Vitoria, 29060-220, Brazil

Email: bocaliniht@hotmail.com

Manuscript received on March 21, 2018

Manuscript accepted on June 1, 2018

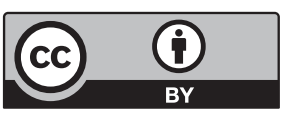

Motriz. The Journal of Physical Education. UNESP. Rio Claro, SP, Brazil - eISSN: 1980-6574 - under a license Creative Commons - Version 4.0 